\title{
Comparison of Outcome between the Implementation of Enhanced Recovery after Surgery Programme (ERAS) vs. Traditional Care, in Elective Abdominal Surgeries
}

\author{
Gopakumar G. ${ }^{1}$, John S. Kurien², Sansho E.U. ${ }^{3}$, Sandeep Abraham Varghese ${ }^{4}$ \\ 1,2,3,4 Department of General Surgery, Government Medical College, Kottayam, Kerala, India.
}

\section{ABSTRACT}

\section{BACKGROUND}

ERAS has been responsible for reducing surgical stress, maintaining postoperative physiological function, and for enhancing mobilization after surgery. It has been ultimately shown to reduce the rates of morbidity, hastening recovery, and reducing the length of hospital stay. The objective of this study was to compare the outcome between the implementation of enhanced recovery after surgery program vs. traditional care in elective abdominal surgeries.

\section{METHODS}

This retrospective study was carried out in the General Surgery Department of Government Medical College, Kottayam, Kerala. This is a tertiary care centre that caters to the needs of nearly five districts in the state. The hospital has excellent critical care and surgical facilities. Patients were grouped as ERAS and Non-ERAS. 78 patients were included in each group. A standard questionnaire was formulated. The clinical outcomes, functional recovery, and patient experience in ERAS and conventional methods of recovery after surgery were analyzed and compared.

\section{RESULTS}

Duration of hospital stay, complications, and costs incurred were found to be almost $60 \%$ less as compared to the non-ERAS group and patient satisfaction was high in the ERAS group. By using ERAS, we have been able to reduce the duration of hospital stay by more than $30 \%$ and the post-operative complications by up to $50 \%$.

\section{CONCLUSIONS}

The ERAS programme has definite advantage over traditional methods in terms of hospital stay, treatment cost, complications, readmission rates and overall patient satisfaction. We have to analyse the avoidable factors leading to substandard care or any missed opportunities. Patient education must also be given utmost importance.

\section{KEY WORDS}

Abdominal Surgeries, Complications, ERAS, Fluid Management, Length of Stay, Nutrition, Patient Satisfaction
Corresponding Author:

Dr. Gopakumar G,

Aishwarya Bhavan,

Nedupuram, Kottukkal

P. O, Kollam - 691306,

Kerala, India.

E-mail:drgopakumar47@gmail.com

DOI: $10.14260 / \mathrm{jemds} / 2020 / 686$

How to Cite This Article:

Gopakumar G, Kurien JS, Sansho EU, et al. Comparison of outcome between the implementation of enhanced recovery after surgery programme (eras) vs. traditional care, in elective abdominal surgeries. J Evolution Med Dent Sci 2020;9(42):31273132, DOI: 10.14260/jemds/2020/686

Submission 02-01-2020,

Peer Review 13-09-2020,

Acceptance 19-09-2020,

Published 19-10-2020.

Copyright (C) 2020 Gopakuamr G. et al. This is an open access article distributed under Creative Commons Attribution License [Attribution 4.0 International (CC BY 4.0)] 


\section{BACKGROUND}

ERAS is a multimodal peri operative pathway designed to achieve early recovery for patients undergoing major surgeries. ERAS operates in the following two ways:

1. Changes the practices traditionally followed to those based on evidence.

2. Comprehensively covers all areas of the patient's journey through the surgical process. ${ }^{1}$

ERAS has been responsible for reducing surgical stress, maintaining postoperative physiological function and enhancing mobilization after surgery. It has been ultimately shown to reduce the rates of morbidity, hastening recovery and reducing the length of hospital stay. ${ }^{2}$

It was to popularize this protocol that the ERAS Society ${ }^{3}$ was founded. It has its roots in what was called the ERAS Study Group, assembled by Professor Ken Fearon and Professor Olle Ljungqvist in 2001 to further develop ideas put forth in the 1990s by Professor Henrik Kehlet. ${ }^{4}$

The ERAS Society discovered that there was a great discrepancy between the actual practices and what was already known to be best practice, based on the literature. This prompted the group to examine the process of change from tradition to best practice. 5

To practice ERAS successfully, there has to be coordination between multiple disciplines, including surgery, anaesthesiology, nursing, nutrition, physical therapy and hospital team members. In this way, the patient will receive well organized care, thus leading to increased effectiveness of clinical management based on the existing evidence. ${ }^{6}$

Colorectal surgery is one of the first surgical modalities where ERAS played a major role. ${ }^{7}$ The components of an enhanced recovery protocol include preoperative patient education, modified preoperative fasting guidelines, preoperative medical optimization, fluid management, the role of bowel preparation, early oral intake, early ambulation and standardized multimodal analgesic regimens. Four areas of patient care pre-operative medical optimization, pain management, use of bowel preparation and choice of intraoperative fluid type are conveniently forgotten to stick to traditional dogmas. The purpose of Enhanced Recovery after Surgery protocols are to shift the paradigm by using the most current evidence to provide new insight into these areas. ${ }^{8}$

Surgery and anaesthesia are dynamic disciplines. Hence there needs to be regular updates of the knowledge base and continuous training of those involved in the treatment of surgical patients. ${ }^{9}$

\section{Objectives}

1. To measure the clinical outcomes of ERAS and conventional methods of recovery after surgery

2. To determine the functional recovery and patient experience in the aforementioned groups

\section{METHODS}

This is a retrospective study carried out in the Department of General Surgery of our institution for nine months from the $1^{\text {st }}$ September 2017 to the $31^{\text {st }}$ May 2018, followed by 3 months post operatively, for each patient including follow up. Based on the sample size calculation the minimum sample size was 78 . Study was conducted among consenting patients undergoing elective abdominal surgeries in the Department of General surgery

\section{Study Procedure}

After getting the Institutional Review Board clearance and approval from the Head of the Department, study was conducted among consenting patients undergoing elective abdominal surgeries in the Department of General surgery, Government Medical College, Kottayam.

Patients who gave consent to take part in the study underwent preoperative, intraoperative and postoperative care as per the standard ERAS guidelines.

Patients were put into identical groups based on whether ERAS guidelines were followed or not. They (the ERAS group) were closely monitored during their pre-operative, intra operative period, followed up in the post-operative period and on subsequent follow up visits and their details entered into a proforma, analysed and statistical significance determined.

\section{ERAS Guidelines ${ }^{3}$}

This was the protocol followed in one group of patients where ERAS was applied-

1. Pre-operative information, education and counsellingdedicated preoperative counselling 10

2. Pre-operative optimization-stop smoking and alcohol consumption four weeks prior to surgery ${ }^{11}$

3. Preoperative preparation of the bowel-not practised routinely in colonic surgeries ${ }^{12}$

4. Preoperative fasting and carbohydrate requirementclear fluids was allowed up to 2 hours and solids up to 6 hours prior to anaesthesia ${ }^{13}$

5. Pre anaesthetic medication-routinely no sedatives were given prior to surgery ${ }^{14}$

6. Prophylaxis against thromboembolism-compression stockings / intermittent pneumatic compression / LMWH $^{15}$

7. Antimicrobial prophylaxis and skin preparation-routine prophylaxis with I / v antibiotics $30-60$ minutes before surgery. skin preparation with povidone iodine. ${ }^{16}$

8. Standard anaesthetic protocol-standard protocol allowed rapid awakening, and the anaesthetist controlled the fluid therapy. ${ }^{17}$

9. Post-operative nausea and vomiting-multimodal approach including Ondansetron, Phenergan etc

10. Laparoscopy and modifications of surgical accesslaparoscopy when expertise was available

11. Nasogastric intubation-not routinely recommended. If inserted during surgery, was removed before reversal of anaesthesia. 18

12. Prevented intraoperative hypothermia-intra op maintenance of body temp > 36 degree centigrade by warming device and warmed IVF19

13. Perioperative fluid management-intra op fluids with optimized cardiac output ${ }^{20}$

14. Drainage of peritoneal cavity after colonic anastomosisdrainage was judiciously done depending on surgeon's decision. 
15. Urinary drainage-transurethral bladder drainage, if done on table, was removed on the next day.

16. Prevention of postoperative ileus by mid thoracic epidural anaesthesia and laparoscopic surgery when possible.

17. Postoperative analgesia-Thoracic epidural anaesthesia using low dose local anaesthetics and opioids.

18. Perioperative nutritional care-active nutritional support was given when undernourished. ${ }^{21}$

19. Postoperative glucose control by avoiding hyperglycaemia and close control with insulin was done when necessary.

20. Early mobilization-to prevent risk of pneumonia / insulin resistance / muscle weakness

In the second group of patients we did not implement the ERAS guidelines. They were also closely monitored during the pre-operative, intra-operative period, and followed up in the post-operative period and on subsequent visits and their details entered into a proforma, analysed and statistical significance determined. ${ }^{22}$

\section{Sample Size}

The minimum sample size required for the study was calculated using the formula

$\mathrm{n}=[(\mathrm{Z} \alpha+\mathrm{Z} \beta)\{\mathrm{P} 1(1-\mathrm{P} 1)+\mathrm{P} 2(1-\mathrm{P} 2)\}] \div(\mathrm{P} 1-\mathrm{P} 2)^{2}$

Using the said equation, the value of ' $\mathbf{n}$ ' was calculated to be 77.45. Hence the minimum sample size required was 78 in each group.

\section{Sampling Technique}

Those patients satisfying inclusion and exclusion criteria were consecutively included in the study till desired sample size was achieved.

\section{Inclusion Criteria}

Patients admitted to the Department of General Surgery for elective abdominal surgeries who were willing to give their consent to take part in the study and of age less than 70 years.

\section{Exclusion Criteria}

- All cases of emergency abdominal surgeries like road traffic accidents.

- Moribund patients.

\section{Statistical Analysis}

Data will be coded and entered in Microsoft excel. Data analysis will be done using SPSS. For qualitative variable frequencies will be calculated \& for quantitative variables mean and SD will be calculated. Association between qualitative variable will be analyzed using chi square test. Association between quantitative variables will be analyzed using independent sample $t$ test. Nonparametric tests will be utilized whenever necessary. Significance level will be fixed at a $\mathrm{p}$ value of $<0.05$.

\section{RESULTS}

The total number of elective abdominal surgeries performed during the one-year study period in the General Surgery Department at Government Medical College, Kottayam was about 850. Of these, 156 cases were studied, 78 of them performed under ERAS in accordance with the inclusion criteria and the rest conventionally.

\section{Age Distribution}

Most patients were $\geq 60$ years of age. There was no significance for age in our study with a p value of 0.333 .

\section{Socio-Economic Status}

Most of the patients in our study belonged to Class II socioeconomic status. There was no significance between the ERAS and non-ERAS groups.

\section{Length of Stay}

The difference between length of stay between the patients who underwent surgery under ERAS protocol and the nonERAS group was found to be highly significant with $\mathrm{p}$ value < 0.05. Maximum number of patients in the ERAS group had a hospital stay of $4-6$ days while maximum number of patients in the conventional care group had to stay in the hospital for $\geq$ 14 days.

\section{Post-Operative Complications}

Post-operative complications, another important variable we considered for this study, was also found to be significantly lower in the ERAS group with a p value of only 0.014. $62.3 \%$ of the patients who underwent surgery under ERAS guidelines did not encounter any complications while the absence of complications for the non-ERAS group was only $37.7 \%$.

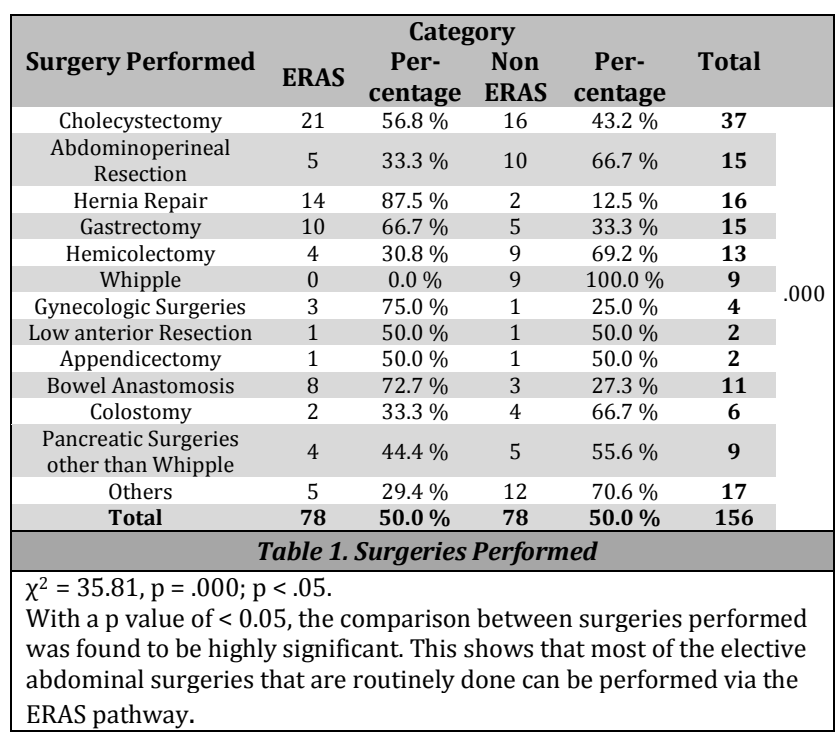




\section{Costs Incurred}

No significant difference was found between the costs incurred between the two groups. This could be due to the fact that ours being a government institution most of the services are provided free or at a subsidized rate. Moreover, there are also several schemes like RSBY for treatment of the poor.

Patient satisfaction, on comparison, was found to be much higher in the ERAS group then the non-ERAS. Almost $60 \%$ patients were satisfied in the ERAS group.

\section{Mode of Surgery}

No significant difference was found between either laparoscopic or open surgeries between the two groups.

\section{Selection of Patients}

78 consecutive elective abdominal surgery patients in S1 unit were studied with ERAS protocol and 78 abdominal surgery patients in all other units were studied with conventional protocol.

\section{Age Groups}

Of the 156 patients we studied, most of them were over the age of 60 years. This was not a variable that was considered pertinent to our study.

\section{Sex}

Almost equal number of males and females were there in each group. This was also not a significant variable.

\section{Socio-Economic Status}

Majority of patients we studied were in the Class II socioeconomic status according to the modified Kuppuswamy scale.

\section{Length of Hospital Stay}

In studies conducted by Krishna K. Vardhan et $\mathrm{al}^{8}$ and U.O Gustaffson et al $^{9}$ it was found that the duration of hospital stay was reduced among the ERAS group significantly as compared to the non-ERAS group. The average stay duration among the various studies are summarized.

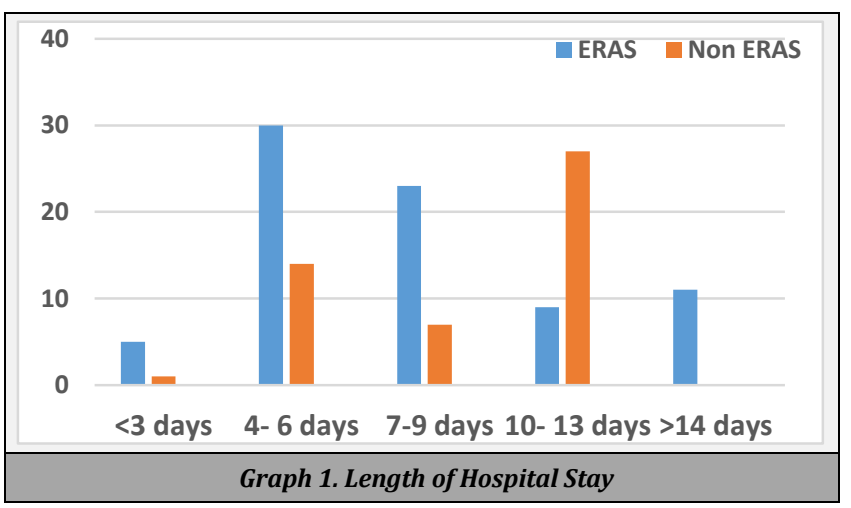

From the table it is evident that our study correlated well with the findings of other international studies with the mean duration of hospital stay in the ERAS group being $4-6$ days. This was found to be a highly significant association with a $\mathrm{p}$ value of $<0.05$.

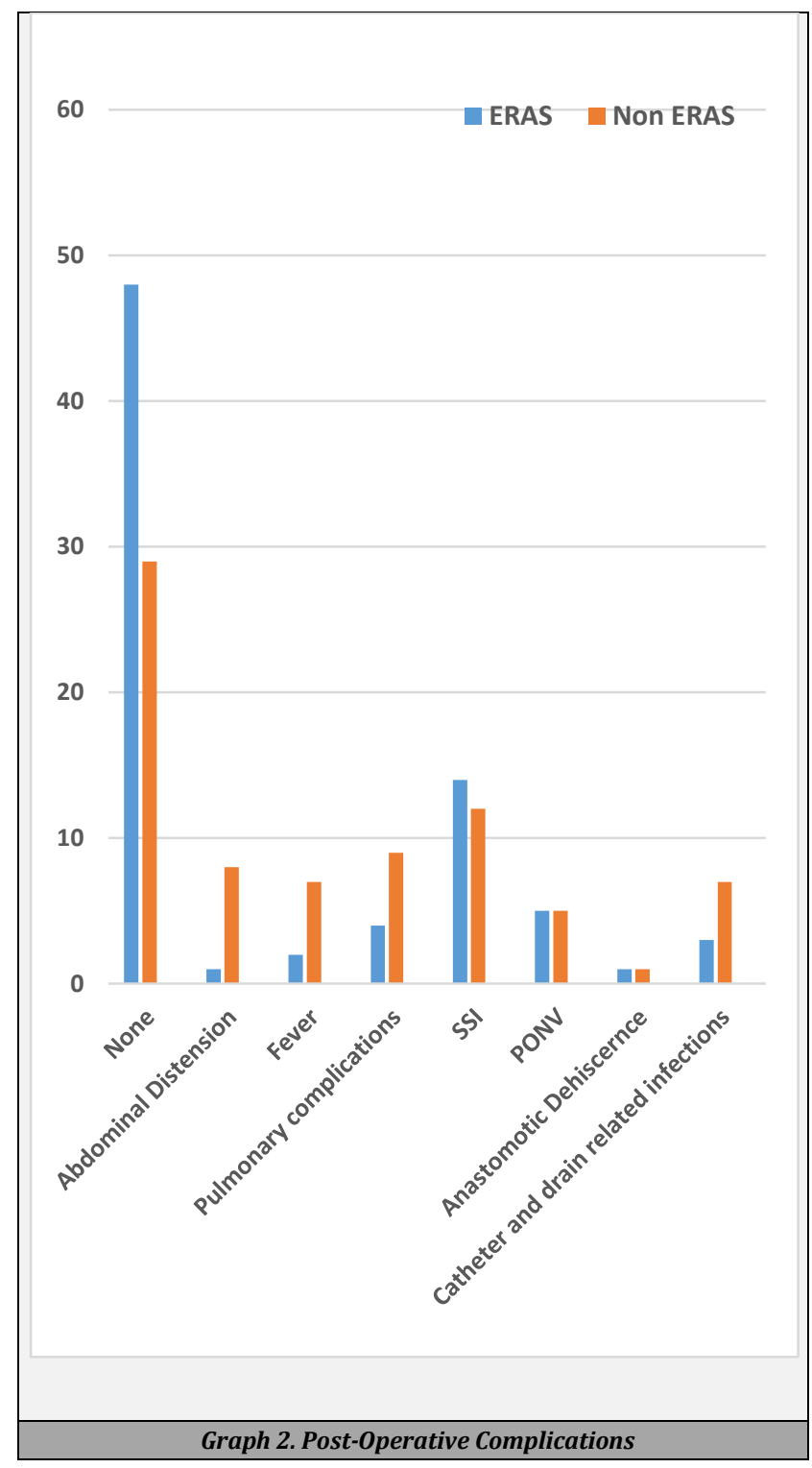

With regard to abdominal surgeries, the major complications considered were abdominal distension, fever, pulmonary complications, surgical site infections, postoperative nausea and vomiting, anastomotic dehiscence and catheter and drain related infections. Just like all other major studies, we also found significantly reduced complications in the ERAS group. The ERAS Society ${ }^{3}$ itself considers that those surgeries that strictly adhere to the ERAS protocols will have $>48 \%$ without complications. From our study it was found that as compared to the traditional group, $62.3 \%$ patients who underwent surgery via ERAS had no complications. The $p$ value was 0.014 .

\section{Costs Incurred}

There was no significant difference among the two groups in the costs incurred. This must probably have been due to the fat 
that most of the patients we studied belonged to the same socioeconomic class. The study setting being a Government hospital, most of the surgeries were performed free or at a subsidized rate. In addition, there were several government aided schemes like RSBY (Rashtriya Swasthya Bhim Yojana) for treatment purpose and the burden of treatment was not borne by the patients' alone.

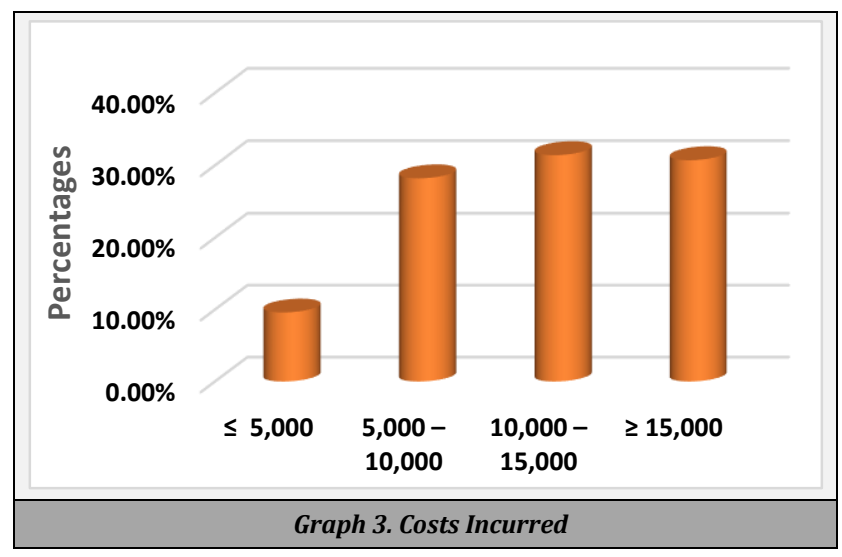

\section{Patient Satisfaction}

Patient satisfaction was assessed on a Likert scale varying on a continuum of extremely dissatisfied being 1 and extremely satisfied being 5. Medline, Embase and Cochrane databases all have extensive studies that confirm the patient satisfaction of ERAS. Our study also found significant association with $p$ value $<0.05$ on comparing patient satisfaction between ERAS and traditional groups.

The major abdominal surgeries performed electively at our center were cholecystectomy, abdominoperineal resection, hernia repair, gastrectomy, hemicolectomy, Whipple, gynecologic surgeries, low anterior resection, appendicectomy, bowel anastomosis, colostomy and other pancreatic surgeries. These were in fact the main abdominal surgeries at any center.

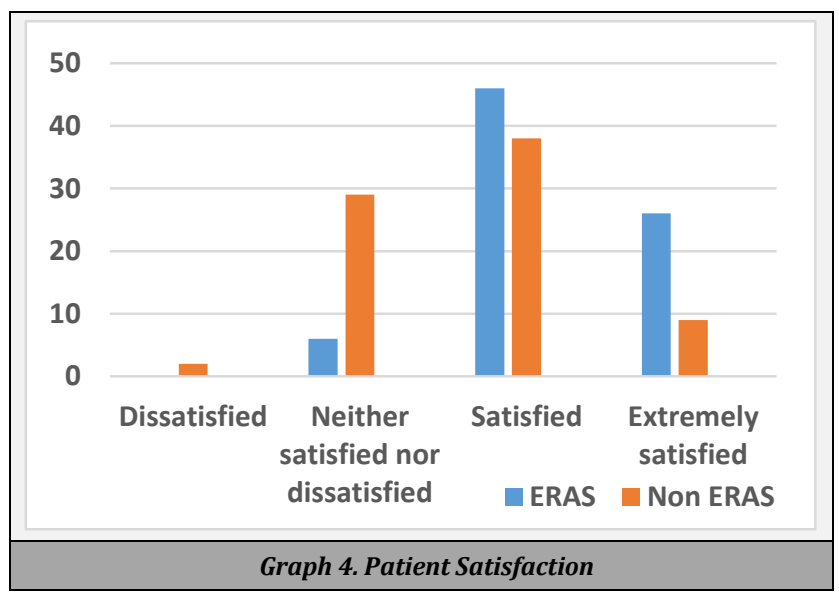

\section{DISCUSSION}

Almost equal number of males and females were there in each group. This was also not a significant variable. Majority of patients we studied were in the Class II socioeconomic status according to the modified Kuppuswamy scale. In studies conducted by Krishna K. Vardhan et al $^{8}$ and U.O Gustaffson et $\mathrm{al}^{9}$ it was found that the length of hospital stay was reduced among the ERAS group significantly as compared to the nonERAS group. The average stay duration among the various studies are summarized. From the table it is evident that our study correlated well with the findings of other international studies with the mean duration of hospital stay in the ERAS group being $4-6$ days. ${ }^{23}$ This was found to be a highly significant association with a $\mathrm{p}$ value of $<0.05$. With regard to abdominal surgeries, the major complications considered were abdominal distension, fever, pulmonary complications, surgical site infections, post-operative nausea and vomiting, anastomotic dehiscence and catheter and drain related infections. Just like all other major studies, we also found significantly reduced complications in the ERAS group. The ERAS Society itself considers that those surgeries that strictly adhere to the ERAS protocols will have > $48 \%$ without complications. From our study it was found that as compared to the traditional group, $62.3 \%$ patients who underwent surgery via ERAS had no complications. The p value was 0.014 . There were no significant differences among the two groups in the costs incurred. This must probably have been due to the fact that most of the patients we studied belonged to the same socioeconomic class.

\section{CONCLUSIONS}

It is of paramount importance that we create an awareness among the surgical and other involved personnel about the benefits of the ERAS protocol. The ERAS programme has definite advantage over traditional methods in terms of hospital stay, treatment cost, complications, readmission rates, and overall patient satisfaction.

ERAS society guidelines ${ }^{24}$ can be routinely applied to all patients undergoing elective abdominal surgeries. If well integrated, we can even extend this to include the emergency surgeries. This has also been extensively studied in orthopedic surgeries.

To successfully integrate Enhanced Recovery into practice, a structured, collaborative, multidisciplinary approach accompanied by education and awareness campaigns may be valuable. It is very important to analyze the avoidable factors leading to substandard care or any missed opportunities. Patient education must also be given utmost importance. As is true with all emerging ideas, the need for further research in this field cannot be over emphasized.

Data sharing statement provided by the authors is available with the full text of this article at jemds.com.

Financial or other competing interests: None.

Disclosure forms provided by the authors are available with the full text of this article at jemds.com.

\section{REFERENCES}

[1] Bernard AC, Daveport DL, Chang PK, et al. Intraoperative transfusion of $1 \mathrm{U}$ to $2 \mathrm{U}$ packed red blood cells is 
associated with increased 30-day mortality, surgical-site infection, pneumonia and sepsis in general surgery patients. J Am Coll Surg 2009;208(5):931-7.

[2] Basse L, Jakobsen DH, Billesbolle P, et al. A clinical pathway to accelerate recovery after colonic resection. Ann Surg 2000;232(1):51-7.

[3] ERAS Compliance Group. The impact of enhanced recovery protocol compliance on elective colorectal cancer resection: results from an international registry. Ann Surg 2015;261(6):1153-9.

[4] Fecher-Jones I, Taylor C, et al. Lived experience, enhanced recovery and laparoscopic colonic resection. Br J Surg 2015;24(4):223-8.

[5] Fleisher LA, Fleischmann KE, Auerbach AD, et al. 2014 ACC/AHA guideline on perioperative cardiovascular evaluation and management of patients undergoing noncardiac surgery: executive summary: a report of the American College of Cardiology/American Heart Association Task Force on Practice Guidelines. Circulation 2014;130(24):2215-45.

[6] Aasvang EK, Luna IE, Kehlet H. Challenges in postdischarge function and recovery: the case of fast track hip and knee arthroplasty. Br J Anaesth 2015;115(6):8616.

[7] Berger R. Now I see it, now I don't: researcher's position and reflexivity in qualitatiove research. Qualitative Research 2015;15(2):219-34.

[8] Varadhan KK, Neal KR, Dejong CH, et al. The enhanced recovery after surgery (ERAS) pathway for patients undergoing major elective open colorectal surgery: a meta-analysis of randomised controlled trials. Clin Nutr 2010;29(4):434-40.

[9] Gustaffson UO, Thorell A, Soop M, et al. Hemoglobin A1c as a predictor of postoperative hyperglycemia and complications after major colorectal surgery. Br J Surg 2009;96(11):1358-64.

[10] Fragiadakis GK, Gaudilliere B, Ganio EA, et al. Patientspecific immune states before surgery are strong correlates of surgical recovery. Anesthesiology 2015;123(6):1241-55.

[11] Artz N, Elvers KT, Lowe CM, et al. Effectiveness of physiotherapy exercise following total knee replacement: systematic review and meta-analysis. BMC Musculoskelet Disord 2015;16:15.
[12] Des Guetz G, Uzzan B, Bouillet T, et al. Impact of physical activity on cancer-specific and overall survival of patients with colorectal cancer. Gastroenterol Res Pract 2013;2013:340851.

[13] Greco M, Capretti G, Beretta L, et al. Enhanced recovery program in colorectal surgery: a meta analysis of randomised control trials. World J Surg 2014;38(6):153141.

[14] American Society of Anesthesiologists Task Force on Acute Pain. Practice guidelines for acute pain management in the perioperative setting: an updated report by the American Anesthesiologists Task Force on Acute Pain Management. Anesthesiology 2012;116(2):248-73.

[15] Hossain FS, Konan S, Patel S, et al. The assessment of outcome after total knee arthroplasty: are we there yet? Bone Joint J 2015;97-B(1):3-9.

[16] Bernard H, Foss M. Patient experiences of enhanced recovery after surgery. Br J Nurs 2014;23(2):100-6.

[17] Jans O, Kehlet H. Postoperative orthostatic intolerance: a common perioperative problem with few available solutions. Can J Anaesth 2017;64(1):10-15.

[18] Cyriac J, Garson L, Schwarzkopf R, et al. Total joint replacement perioperative surgical home program: 2year follow-up. Anesth Analg 2016;123(1):51-62.

[19] Jorgensen CC, Petersen MA, Kehlet H. Preoperative prediction of potentially preventable morbidity after fasttrack hip and knee arthroplasty: a detailed descriptive cohort study. BMJ Open 2016;6(1):e009813.

[20] Dahlke AR, Merkow RP, Ching JW, et al. Comparison of postoperative complication risk prediction approaches based on factors known preoperatively to surgeons versus patients. Surgery 2014;156(1):39-45.

[21] Hendry PO, Hausel J, Nygren J, et al. Determinants of outcome after colorectal resection within an enhanced recovery programme. Br J Surg 2009;96(2):197-205.

[22] Joint Commission. Implementation guide for the Joint Commission patient blood management measures, 2011. www.jointcommission.org/assets/1/6/PBM Implementation_Guide_20110624.pdf.

[23] Braga M, Ljungqvist O, Soeters P, et al. ESPEN Guidelines on parenteral nutrition: surgery. Clin Nutr 2009;28(4):378-86.

[24] ERAS Society Website. www.erassociety.org (Last accessed on 11/06/2017) 